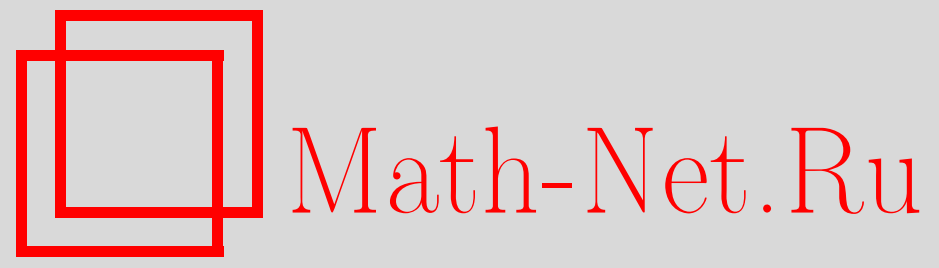

В. П. Костов, Конфигурации корней для гиперболических многочленов степени 3,4 и 5 , Функи. анализ и его прил., 2002, том 36, выпуск 4, 71-74

DOI: https://doi.org/10.4213/faa221

Использование Общероссийского математического портала MathNet.Ru подразумевает, что вы прочитали и согласны с пользовательским соглашением

http://www . mathnet.ru/rus/agreement

Параметры загрузки:

IP: 3.89 .197 .203

26 апреля 2023 г., 07:57:44




УДК 512.622

\title{
Конфигурации корней для гиперболических многочленов степени 3,4 и 5
}

\author{
(c) 2002. В. П. Костов
}

\section{Посвящается памяти моей матери}

1. Введение. Вещественный многочлен степени $n$ одной вещественной переменной называется гиперболическим (соответственно строго гиперболическим), если все его корни вещественные (соответственно вещественные и разные). Производные всех порядков, не превосходящих $n-1$, (строго) гиперболического многочлена (строго) гиперболичны. Если коэффициенты многочлена зависят от параметров, то множество наборов значений параметров, для которых многочлен имеет только вещественные корни, называется областью гиперболичности. Обозначим через $x_{j}^{i}$ корни $i$-й производной гиперболического многочлена степени $n, x_{1}^{i} \leqslant \cdots \leqslant x_{n-i}^{i}$. Из стандартной теоремы Ролля вытекает, что для $k>i$, $j=1, \ldots, n-k$ имеют место неравенства

$$
x_{j}^{i} \leqslant x_{j}^{k} \leqslant x_{j+k-i}^{i}
$$

со строгими неравенствами для строго гиперболического многочлена. Рассматривается вопрос, какие невырожденные конфигурации (т. е. конфигурации, удовлетворяющие условию (1) без равенств вида $x_{j_{1}}^{i_{1}}=x_{j_{2}}^{i_{2}}$ для каких-либо $\left(i_{1}, j_{1}\right) \neq$ $\left.\left(i_{2}, j_{2}\right)\right)$ реализуются корнями гиперболического многочлена и его производных. Для $n=1,2$ или 3 все такие конфигурации реализуемы, но для $n=4$ две из 12 конфигураций не реализуются гиперболическими многочленами, см. разд. 2 (но они реализуются негиперболическими многочленами степени выше 4).

ТЕОРема 1. Имеются ровно 116 невырожденных конфигураций, которые реализуются корнями гиперболического многочлена степени 5 и его производных порядка не выше 4.

Эта теорема и остальные результаты настоящей заметки доказаны в [5].

С другой стороны, имеются 286 невырожденных конфигураций, удовлетворяющих условию (1) (для любого $n$ это число равняется $\left(C_{n+1}^{2}\right) ! \frac{1 ! 2 ! \ldots(n-1) !}{1 ! 3 ! \ldots(2 n-1) !}$, см. [11] или [12]). Отсутствие некоторых конфигураций тесно связано с присутствием переопределенных стратов в любом семействе общего положения многочленов степени $\geqslant 4$ со старшим коэффициентом 1 (см. разд. 3 ).

В [8] показано, что все конфигурации, удовлетворяющие условию (1), состоящие из корней гиперболического многочлена и одной из его производных, реализуются. Это неверно для конфигураций, состоящих из корней двух производных гиперболического многочлена (см. замечание 3). Изучение гиперболических многочленов было начато в работах $[2-4,6,7]$. Некоторые результаты были получены параллельно в статьях [9] и [10]; случай $n=4$ упоминается в [1]. Рассматриваемые здесь проблемы сформулированы В. И. Арнольдом и Б. З. Шапиро; они же привлекли к этим проблемам внимание автора. Автор выражает им свою самую искреннюю благодарность.

2. Случаи $\boldsymbol{n}=\mathbf{2}$ и 3 . Рассмотрим семейство общего положения многочленов $P(x, a)=x^{n}+a_{1} x^{n-1}+\cdots+a_{n}, a_{i} \in \mathbb{R}$. Обозначим через $\Pi^{*}$ его область 
гиперболичности. Можно положить $a_{1}=0$, чего можно добиться сдвигом переменной $x$. Следовательно, $P$ будет гиперболическим лишь при $a_{2} \leqslant 0$ и при $a_{2}=0$ он будет гиперболическим, только если $a_{2}=\cdots=a_{n}=0$. Положим (для $t \in \mathbb{R}) \tilde{a}_{j}=e^{j t} a_{j}$. Имеет место равенство $P\left(e^{t} x, \tilde{a}\right)=e^{n t} P(x, a)$. Вот почему изучение $\Pi^{*}$ сводится к изучению $\Pi=\left.\Pi^{*}\right|_{a_{1}=0, a_{2}=-1}$. Чтобы изучить возможные невырожденные конфигурации корней многочлена $P$ и его производных, можно изучить дискриминанты $D(i, j)=\left\{\left(a_{2}, \ldots, a_{n}\right) \in \Pi \mid \operatorname{Res}\left(P^{(i)}, P^{(j)}\right)=0\right\}$. Для $n=4$ и 5 это сделано в [5].

Для $n \leqslant 5$ обозначим корни многочлена $P$ (соответственно $\left.P^{\prime}, P^{\prime \prime}, P^{\prime \prime \prime}, P^{(4)}\right)$ через $x_{1} \leqslant \cdots \leqslant x_{5}$ (соответственно через $f_{1} \leqslant \cdots \leqslant f_{4}, s_{1} \leqslant s_{2} \leqslant s_{3}, t_{1} \leqslant t_{2}, l$ ). Для $n=3$ рассмотрим семейство $P(x, a)=x^{3}-x+a$. Тогда $\Pi=[-2 \sqrt{3} / 9,2 \sqrt{3} / 9]$, $D(0,1)=\{ \pm 2 \sqrt{3} / 9\}, D(0,2)=\{0\}$. Для $a<0, a \in \Pi$ (соответственно $a>0$, $a \in \Pi$ ) справедливы неравенства $x_{1}<f_{1}<x_{2}<s_{1}<f_{2}<x_{3}$ (соответственно $\left.x_{1}<f_{1}<s_{1}<x_{2}<f_{2}<x_{3}\right)$.
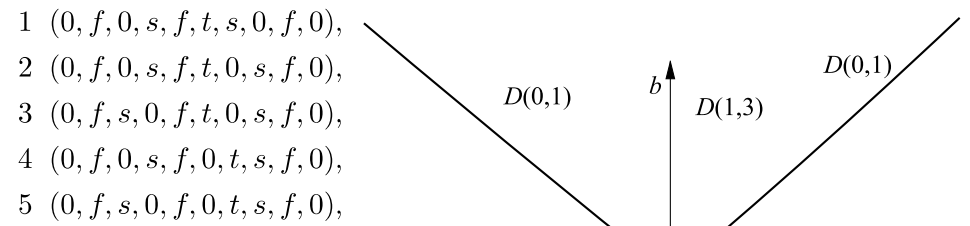

$6(0, f, 0, s, t, f, s, 0, f, 0)$, $7(0, f, s, 0, t, f, s, 0, f, 0)$, $8(0, f, s, 0, t, f, 0, s, f, 0)$, $9(0, f, s, t, 0, f, s, 0, f, 0)$, $10(0, f, s, t, 0, f, 0, s, f, 0)$,

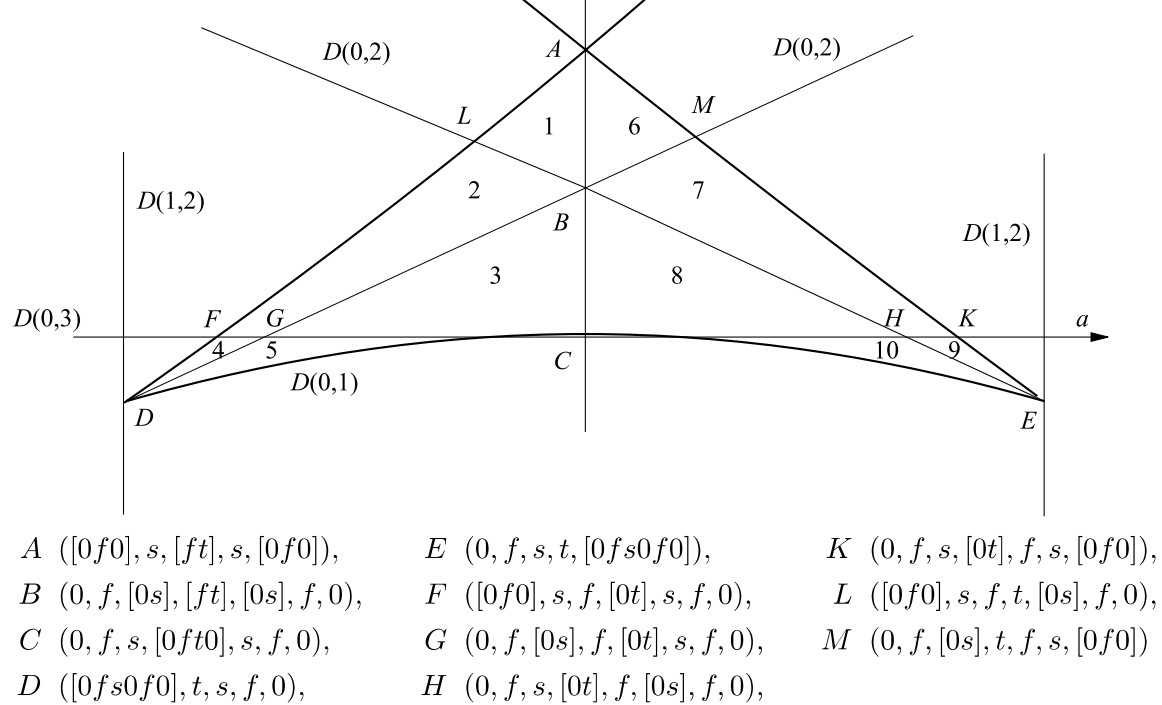

Рис. 1. Случай $n=4$

Рассмотрим для $n=4$ семейство $P(x, a, b)=x^{4}-x^{2}+a x+b, x, a, b \in \mathbb{R}$. Областью гиперболичности П семейства $P$ является замыкание внутренности криволинейного треугольника $D E A$, имеющего точки возврата $D$ и $E$, см. рис. 1. Конфигурации корней многочленов $P, P^{\prime}, P^{\prime \prime}$ и $P^{\prime \prime \prime}$ (обозначенных соответственно через $0, f, s, t)$ указаны возле рисунка; совпадающие корни указаны в квадратных скобках. Согласно общей формуле, приведенной во введении, имеются 12 невырожденных конфигураций, удовлетворяющих (1). Отсутствие двух конфигураций связано с тем, что $D(1,3)$ проходит через точку пересечения двух прямых, из которых состоит $D(0,2)$. 
3. Переопределенные страты. Обозначим через $\mathrm{Pol}_{n}^{\mathbb{C}}$ (соответственно $\mathrm{Pol}_{n}$ ) пространство всех многочленов степени $n$ с комплексными коэффициентами и со старшим коэффициентом 1 (соответственно всех гиперболических многочленов степени $n$ со старшим коэффициентом 1$)$. Множество всевозможных наборов многочленов $\left(P_{n}, \ldots, P_{1}\right)$, где $P_{i} \in \mathrm{Pol}_{i}^{\mathbb{C}}$ (соответственно $P_{i} \in \mathrm{Pol}_{i}$ ), можно отождествить с пространством $\widetilde{X}$ корней этих многочленов (мы обозначаем корни многочлена $P_{i}$ через $x_{j}^{i}$; в случае $\mathrm{Pol}_{n}$ выполняются неравенства $\left.x_{1}^{i} \leqslant \cdots \leqslant x_{n-i}^{i}\right)$. Стратифицируем $\widetilde{X}$. Страт коразмерности $k-$ это подмножество пространства $\widetilde{X}$, определяемое $k$ независимыми равенствами вида $x_{j_{1}}^{i_{1}}=x_{j_{2}}^{i_{2}}$, $\left(j_{1}, i_{1}\right) \neq\left(j_{2}, i_{2}\right)$. Для $P \in \operatorname{Pol}_{n}^{\mathbb{C}}$ или $P \in \operatorname{Pol}_{n}$ положим $P^{n-i}=((n-i) ! / n !) P^{(i)}$. Можно вложить $\operatorname{Pol}_{n}$ в $\widetilde{X}$, отображая $P \in \operatorname{Pol}_{n}$ в $\left(P^{n}, \ldots, P^{1}\right)$; обозначим это вложение через $\pi$. Для страта $S \in \widetilde{X}$ обозначим через $S^{\prime}$ его пересечение с $\pi\left(\mathrm{Pol}_{n}\right)$. Страт $S$ называется переопределенным, если его коразмерность в $\widetilde{X}$ (называемая его порядком, ord $S$ ) больше, чем коразмерность множества $S^{\prime}$ в $\pi\left(\mathrm{Pol}_{n}\right)$ (называемая его коразмерностью, $\left.\operatorname{codim} S\right)$. Множество $S^{\prime}$, когда оно непусто, называется стратом области П (переопределенным стратом, если страт $S$ переопределенный).

В случае $n=5$ рассмотрим семейство $P=x^{5}-x^{3}+a x^{2}+b x+c$. Множества П и $D(i, j)$ инвариантны относительно инволюции $\sigma:(a, b, c) \mapsto(-a, b,-c)$.

Теорема 2. Для $n<4$ не существует переопределенных стратов. Для $n=4$ точки А и В (см. рис. 1) определяют единственные переопределенные страты области П. Для $n=5$ единственные переопределенные страты множества $\Pi$ - это точки $\Sigma, \Phi, F \in D(2,4)$, где

$$
\begin{array}{ll}
\Sigma: x_{2}=t_{1}, x_{4}=t_{2}, x_{3}=s_{2}=l, & \operatorname{ord} \Sigma=4, \operatorname{codim} \Sigma=3, \\
\Phi: x_{2}=s_{1}, x_{4}=s_{3}, x_{3}=s_{2}=l, & \operatorname{ord} \Phi=4, \operatorname{codim} \Phi=3, \\
F: x_{1}=x_{2}, x_{4}=x_{5}, x_{3}=s_{2}=l, f_{2}=t_{1}, f_{3}=t_{2}, & \operatorname{ord} F=6, \operatorname{codim} F=3 .
\end{array}
$$

ЗАмЕчАНИЕ 3 . Для $n=5$ следующая конфигурация (удовлетворяющая (1)) корней производных $P^{\prime}$ и $P^{\prime \prime \prime}$ не реализуется: $f_{1}<f_{2}<t_{1}<t_{2}<f_{3}<f_{4}$. Ее отсутствие тесно связано с наличием переопределенного страта, определяемого точкой $F$ из теоремы 2 , см. [5].

ОПРЕДЕЛЕнИЕ 4. Вектором кратности называется вектор, компоненты которого являются кратностями корней гиперболического многочлена. Пример: $[1,2,2]$ означает $x_{1}<x_{2}=x_{3}<x_{4}=x_{5}$.

ПримеР 5 . Для четного $n \geqslant 4$ (соответственно нечетного $n \geqslant 5$ ) многочлен $P_{e}=\left(x^{2}-2 / n\right)^{n / 2}$ (соответственно $\left.P_{o}=x\left(x^{2}-2 /(n-1)\right)^{(n-1) / 2}\right)$ определяет переопределенный страт. Действительно, $P_{e}^{(n-2)}$ делит $P_{e}^{\prime \prime}$. С другой стороны, многочлен $P_{e}$ полностью определяется тем, что его вектор кратности равняется $[n / 2, n / 2]$ и что его первые три коэффициента равняются 1,0 и -1 ; из этих условий формально не вытекает, что $P_{e}^{(n-2)}$ делит $P_{e}^{\prime \prime}$. Верно также, что $P_{e}^{(n-1)}=n ! x$ делит $P_{e}^{(k)}$, если $k$ нечетно. Для $P_{o}$ верно, что $P_{o}^{(n-2)}$ делит $P_{o}^{\prime}$. Однако $P_{o}$ полностью определяется тем, что его вектор кратности равняется $[(n-1) / 2,1$, $(n-1) / 2]$, что $P_{o}$ делится на $P_{o}^{(n-1)}=n ! x$ и что первые его три коэффициента равняются 1,0 и -1 ; в эти условия не входят ни $P_{o}^{(n-2)}$, ни $P_{o}^{\prime}$. Для $n=4$ и 5 многочлены $P_{e}$ и $P_{o}$ определяются точками $A$ и $F$ (см. теорему 2$)$. 
К. ван дер Мей, В. Н. Пивоварчик

ОПРЕДЕЛЕНИЕ 6. Многочленом Гегенбауера степени $n$ называется гиперболический многочлен вида $x^{n}-x^{n-2}+\ldots$, который делится на свою вторую производную (для $n \geqslant 3$ существует единственный такой многочлен; он является четным при четном $n$ и нечетным при нечетном $n$ ). Следовательно, для $n \geqslant 4$ многочлен Гегенбауера определяет переопределенный страт размерности 0 в семействе $\left.P\right|_{a_{1}=0, a_{2}=-1}$, поскольку он полностью определяется тем, что делится на свою вторую производную, а имеется дополнительное условие, что $P^{(n-1)}=n ! x$ делит все его производные, являющиеся многочленами нечетной степени.

Для $n=3$ (соответственно $n=4, n=5$ ) многочлен Гегенбауера равняется $x^{3}-x$ (соответственно $x^{4}-x^{2}+5 / 36$ (см. точку $B$ на рис. 1 ), $x^{5}-x^{3}+21 x / 100$ (см. точку $\Phi$ в теореме 2$)$ ). Интегрируя многочлены, задающие переопределенные страты, можно получать переопределенные страты более высокой размерности в

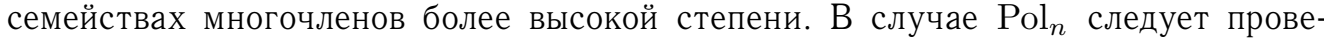
рить, для каких значений постоянной интегрирования получаемый многочлен гиперболичен.

\title{
ЛИТЕРАТУРА
}

1. Anderson B. Amer. Math. Monthly, 100, 864-866 (1993). 2. Арнольд В. И. Функц. анализ и его прил., 20, вып. 2, 52-53 (1986). 3. Арнольд В. И. Труды Тбилисск. унив., 232/233, 23-29 (1982). 4. Givental A. B. УМH, 42, вып. 2, 221-222 (1987). 5. Kostov V. P. Serdica Math. J., 28, No. 2, 117-152 (2002); Prépublication No. 620 de l'UNSA, juin 2001. 6. Kostov V. P. Proc. Roy. Soc. Edinb., 112, No. 3-4, 203-211 (1989). 7. Kostov V. P. Serdica Math. J., 25, No. 1, 47-70 (1999). 8. Kostov V. P., Shapiro B. Z. Bull. Sci. Math., Vol. 126 No. 1, pp. 45-60 (January 2002); Prépublication No. 19 de l'Université de Nice, Juin 2001. 9. Meguerditchian I. Math. Z, 211, 449-460 (1992). 10. Meguerditchian I. Géométrie du discriminant réel et des polynômes hyperboliques. Thèse de doctorat, Univ. de Rennes I, 1991. 11. Ruskey F. J. Comb. Theory, 52,77-101 (1992). 12. Thrall M. Michigan Math. J., 1, 81-88 (1952).

Université de Nice-Sophia Antipolis,

Laboratoire de Mathématiques

Поступило в редакцию e-mail: kostov@math.unice.fr

12 ноября 2001 г.

УДК $517.43+517.9$

\section{Обратная задача Штурма-Лиувилля с зависящими от спектрального параметра краевыми условиями*}

\author{
(C) 2002. К. ВАН ДЕР МЕЙ, В. Н. ПИВОВАРЧИК
}

В [1] была рассмотрена следующая краевая задача:

$$
\begin{aligned}
y^{\prime \prime}+\lambda^{2} y-i \lambda p y-q y & =0, \\
y(0) & =0, \\
y^{\prime}(a)+\left(-m \lambda^{2}+i \alpha \lambda+\beta\right) y(a) & =0,
\end{aligned}
$$

где $\lambda-$ спектральный параметр, $a>0, p>0, m>0, \alpha>0, \beta \in \mathbb{R}$, а вещественная функция $q(x)$ принадлежит пространству Соболева $W_{2}^{2}(0, a)$. Задача о

* Исследования первого автора выполнены при частичной финансовой поддержке INdAM-GNCS и MURST, а второго - при частичной финансовой поддержке Автономной области Сардинии. 